\title{
Estimation of Coherence Between Blood Flow and Spontaneous EEG Activity in Neonates
}

\author{
David M. Simpson*, Member, IEEE, Daniel A. Botero Rosas, and Antonio Fernando C. Infantosi
}

\begin{abstract}
Blood flow to the brain responds to changes in neuronal activity and, thus, metabolic demand. In earlier work, we observed correlation between cerebral blood flow and spontaneous electroencephalogram (EEG) activity in neonates. Using coherence, we now found that during Tracé Alternant EEG activity in quiet sleep of normal term neonates, this correlation is strongest at frequencies around $0.1 \mathrm{~Hz}$, reaching statistical significance $(p<0.05)$ in six of the nine subjects studied $(p<0.07$ in eight subjects). Due to noise, artifact, and spontaneous changes in the subjects' EEG patterns, the signals investigated included epochs of missing samples. We, therefore, developed a novel algorithm for the estimation of coherence in such data and applied a Monte Carlo (surrogate data) method for its statistical analysis. This process provides a test for the statistical significance of the maximum coherence within a selected frequency band. In addition to permitting further insight into the mechanisms of cerebral blood flow control, these algorithms are potentially of great benefit in a wide range of biomedical applications, where interrupted (gapped) recordings are often a problem.
\end{abstract}

Index Terms-Cerebral blood flow, coherence, electroencephalogram (EEG), missing samples, Monte Carlo methods, neonates.

\section{INTRODUCTION}

$\mathbf{I}^{1}$ $\mathrm{T}$ has long been known that there is a link between the metabolic activity of the brain and cerebral blood flow [1], [2]. Blood flow to the brain changes, for example, with sleep [3], [4], during epileptic seizures [5], [6], and with sensory stimulation [7]. In previous work [8], we investigated the correlation between cerebral blood flow velocity (CBFV), as measured by transcranial Doppler ultrasound, and spontaneous electroencephalogram (EEG) activity during quiet sleep in newborn babies [Tracé Alternant (TA) EEG pattern]. We found low, but significant, correlation between the signals, with changes in CBFV lagging those in the EEG by about $5 \mathrm{~s}$. In this paper, we extend that work by investigating the coherence between these signals. The coherence gives the correlation between signals selectively for frequency bands, and its use has become particularly widespread in the analysis of neurophysiological signals.

As in the previous work, the quality of the signals available posed a significant technical challenge and required the devel-

Manuscript received October 31, 2003; revised October 10, 2004. This work was supported by Wellcome Trust under Project 064701/Z/01/Z and by the Brazilian agencies CNPq and CAPES. Asterisk indicates corresponding author.

*D. M. Simpson is with the Institute of Sound and Vibration Research, University of Southampton, SO17 1BJ Southampton, U.K. (e-mail: ds@isvr.soton.ac.uk).

D. A. Botero Rosas and A. F. C. Infantosi are with the Biomedical Engineering Program, Federal University of Rio de Janeiro (COPPE/UFRJ), 21945-970 Rio de Janeiro, Brazil (e-mail: dany@ peb.ufrj.br; afci@ peb.ufrj.br). Digital Object Identifier 10.1109/TBME.2005.845368 opment of new methods for estimating coherence and testing its statistical significance. It is difficult to obtain high-quality EEG and CBFV signals for prolonged periods (say, $10 \mathrm{~min}$ ). When the baby moves, the signals are often lost or severely degraded by artifacts and noise. In addition, the EEG pattern of interest, TA, is usually only intermittently present, and thus, the signals analyzed are interrupted by periods of missing data. Most standard signal processing algorithms cannot deal with such cases. Such incomplete data are a common problem in biomedical signal processing, and they are usually dealt with by either analyzing the largest possible segment of good data (often discarding smaller segments) or interpolation (when only short segments are missing). Neither method is suitable in the present study and we, therefore, sought to develop an estimator of coherence that does not require uninterrupted recordings. Furthermore, a Monte Carlo approach to determining the statistical significance of coherence estimates, and in particular the maximum coherence in a given frequency band, is also described.

The methods were developed specifically for the investigation of the coherence between CBFV and EEG, but we believe they are also of great relevance in many other biomedical engineering applications. A range of signal processing methods that can deal with missing samples have been discussed in the recent signal processing literature [9]-[11], but so far, these methods have not been extensively employed in biomedical applications. The development and application of such methods is timely, because they allow for a more effective use of any signals recorded from patients, which is highly desirable from both economic and ethical considerations.

In Section II, we will first describe the signals acquisition and then the algorithms for estimating coherence and the Monte Carlo statistical significance tests. The results of simulation studies to test the novel algorithms are then shown, followed by results from signals recorded in neonates. Some implications of the current work, problems, and future developments are discussed in the Section IV.

\section{MATERIALS AND METHODS}

\section{A. Data Acquisition and Preprocessing}

The set of signals used are the same as in the previous work [8]. A group of nine normal newborns (gestational age 37-41 weeks, Apgar $\geq 8$ at 1 and $5 \mathrm{~min}$ ) were studied within seven days of birth (six of them within the first three days), after written informed parental consent and with approval of the local ethics committee (Instituto Fernandes Figueira, Fiocruz, Rio de Janeiro, Brazil). With the babies asleep, generally after feeding, transcranial Doppler ultrasound signals were acquired from the right middle cerebral artery (MCA) using a $9.56-\mathrm{MHz}$ 
continuous wave system (Parks Electronics Lab., 806FC) and a carefully positioned hand-held pencil-probe. The system's audio output was fed into a purpose-built hardware/software system developed at the Leicester Royal Infirmary, Leicester, U.K. This system estimated the maximum frequency envelope to provide CBFV and simultaneously digitized the EEG (derivation F4-T4), from a polygraphy system (EEG-5414K, Nihon Kohden). The fronto-temporal EEG derivation was chosen because it overlies the cortical region irrigated by the MCA. The EEG signal was acquired with the standard filter setting of 0.5 to $70 \mathrm{~Hz}$. Both the CBFV and the EEG signals were sampled at $200 \mathrm{~Hz}$.

EEG signals were simultaneously recorded on paper (10 lead montage, International 10-20 System, modified for newborns) and then visually analyzed by experienced clinical staff. Based on the complementary signals of electrooculogram (EOG), electromyogram (EMG) (submentalis), and electrocardiogram (ECG) (derivation D2), epochs of EEG showing the TA pattern of EEG, free of excessive noise or artifact, were identified. The corresponding segments of digitized signals (EEG and $\mathrm{CBFV}$ ) were then found. In the raw CBFV signals, spikes were removed and the signals were low-pass filtered (zero phase) with a cutoff frequency of $20 \mathrm{~Hz}$. The EEG signals were also low-pass filtered (35-Hz cutoff). In the EEG signals, the power in the frequency band from approximately 0.5 to $8.5 \mathrm{~Hz}$ was calculated in consecutive intervals of $1 \mathrm{~s}$, using a discrete Fourier transform and a rectangular (boxcar) window. The mean CBFV was calculated for each heartbeat, from which the average CBFV in each 1-s interval was found (in the neonates, a 1-s period typically corresponds to two to three heartbeats). Periods of signals evidently contaminated by strong artifacts (evident large movement artifacts in the raw EEG, or dropout and loss of a pulsatile signal in the raw CBFV) were identified by experienced technicians and excluded from further analysis, by marking them "missing data." An example of the resulting mean CBFV (mCBFV) and the square-root of the power of the EEG (rmsEEG) signals are shown in Fig. 1. These signals were decimated to a $1-\mathrm{Hz}$ sampling rate, after anti-alias filtering ( $0.45-\mathrm{Hz}$ cutoff, zero phase). The periods of missing samples were marked in both signals such that all gaps coincide, although this is not required in the analysis methods presented. From the nine subjects studied, a total of 12 such signals were obtained for analysis (Table I); i.e., three subjects contributed two recordings. The length and the fraction of missing samples are also given in Table I.

\section{B. The Algorithm for Estimating Coherence}

The coherence is defined as

$$
C(f)=\frac{P_{x y}(f)}{\sqrt{P_{x x}(f) P_{y y}(f)}}
$$

where $P_{x x}(f)$ and $P_{y y}(f)$ are the power spectral densities of the digital signals $x[i]$ and $y[i]$, respectively, and $P_{x y}(f)$ is their cross-spectrum. The most common method of estimating coherence uses overlapped fast Fourier transforms (FFT) [12] to find the auto- and cross-spectra. Clearly, this approach is not feasible in the current signals, as the FFT requires all samples in each window to be available.
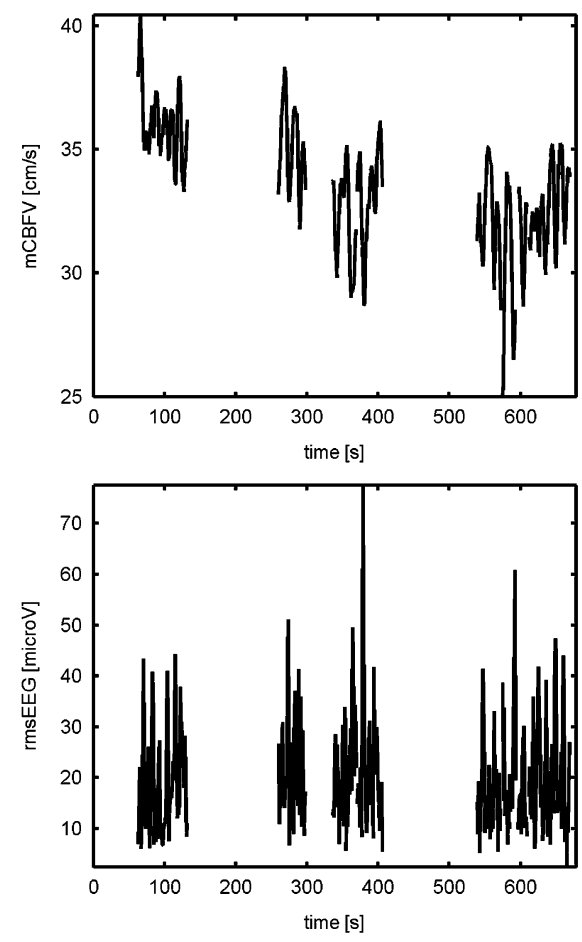

Fig. 1. An example of the mCBFV signal and the root-mean-square EEG (rmsEEG) signals. In this case, $50 \%$ of the data is missing, and the same segments of data have been marked as missing in both signals.

From the Wiener-Khintchine theorem [13], the spectra are given by the discrete Fourier transform (DFT) of the auto- and cross-correlation functions, respectively. This formed the basis of the usual approach to spectral estimation, before the Welch spectral estimates (using overlapped, windowed FFTs) [14] became popular, and is the approach adopted here. The cross-correlation functions can be estimated as

$$
\hat{R}_{x y}[m]=\frac{1}{N-m} \sum_{i=0}^{N-m-1} x[i] y[i+m]
$$

where $m$ is the lag, and indicates an estimate. This is known as the "unbiased estimate," as the sum is normalized by the number of samples for which the product $x[i] y[i+m]$ could be evaluated. In the case of missing samples, this approach may be generalized, and

$$
\hat{R}_{x y}[m]=\frac{1}{L} \sum_{i} x[i] y[i+m]
$$

where $i \in l, l$ being the set of samples (numbering $L$ in total), where neither $x[i]$ nor $y[i+m]$ are missing; $l$, thus, is not a continuous sequence, but it contains gaps. $L$ evidently depends on the length of the data, the lag $m$, and the pattern of missing samples. For the case of no missing samples, (2) and (3) are exactly equivalent. In a similar way, the autocorrelation functions $\hat{R}_{x x}[m]$ and $\hat{R}_{y y}[m]$ can be found. We are, thus, proposing that to estimate the coherence, we first estimate the auto- and cross-correlation functions, and then DFT these to obtain the estimate of the auto- and cross-spectra required in (1).

In estimating the auto- and cross-spectra using the Welch-method, Hanning data-windows are probably most commonly applied to control spectral leakage. Applying a 
TABLE I

The Total Length of the Data AND the PERCENTAGe of Missing SAMPLES IN THE mCBFV AND rmsEEG SignALS For EACH OF THE 12 RECORDINGS

\begin{tabular}{|c|c|c|c|c|c|c|c|c|c|c|c|c|}
\hline Subject (Recording) & $1(1)$ & $1(2)$ & 2 & 3 & $4(1)$ & $4(2)$ & 5 & 6 & $7(1)$ & $7(2)$ & 8 & 9 \\
\hline Length of recording (s) & 354 & 280 & 586 & 496 & 76 & 607 & 368 & 120 & 496 & 190 & 433 & 117 \\
\hline $\begin{array}{l}\text { Samples } \\
\text { missing (\%) }\end{array}$ & 36.2 & 52.1 & 46.4 & 54.8 & 9.2 & 50.1 & 11.4 & 19.2 & 1.0 & 10.5 & 20.1 & 0 \\
\hline$p(\max$. coherence) & 0.637 & 0.349 & 0.022 & 0.008 & 0.040 & $<0.002$ & $<0.002$ & 0.052 & $<0.002$ & 0.002 & $<0.002$ & 0.060 \\
\hline
\end{tabular}

window to the signal is equivalent (in terms of the expected value of the spectral estimate) to applying the same window, convolved with itself, to the auto- (or cross-) correlation function [13]. The window applied to the auto- (or cross-) correlation function is known as the lag-window.

For the cross spectrum, therefore

$$
\hat{P}_{x y}(f)=\operatorname{DFT}\left\{\hat{R}_{x y}[m] \cdot w[m]\right\} / f_{s}
$$

where $\hat{R}_{x y}[m]$ is obtained in accordance with (3), $w[m]$ is the lag-window, and $f_{s}$ is the sampling rate. An equivalent estimator is then used for the auto-spectra. Finally, the coherence estimate is found as

$$
\hat{C}(f)=\frac{\hat{P}_{x y}(f)}{\sqrt{\hat{P}_{x x}(f) \hat{P}_{y y}(f)}} .
$$

In conventional coherence estimates, overlapped windows are employed, and auto- and cross-spectra are obtained by averaging over these windows, to reduce estimation errors. In the algorithm presented here, we follow the correlogram method [13] of spectral estimation, where the spectra are smoothed by applying a lag-window to the correlation functions. As with conventional coherence estimates, the length of the window represents a compromise between frequency resolution (which increases with longer windows) and random errors (which decrease when a larger number of windows is employed). In the current application, we selected data-windows of length $26 \mathrm{~s}$ (corresponding to a frequency resolution of approximately 0.04 $\mathrm{Hz}$ ), which corresponds to lag-windows of 51 samples. The selected frequency resolution was considered the minimum necessary for the range of frequencies of interest (up to $0.5 \mathrm{~Hz}$ ). Although a higher frequency resolution would be desirable, this would require longer lags. However, it is known that correlation estimates become progressively less robust at longer lags [13], and this is exacerbated when some samples are missing. The auto- and cross-spectra were obtained by zero-padding to 64 samples before applying the DFT in (4), such that coherence estimates are found at intervals of $0.016 \mathrm{~Hz}$.

\section{The Algorithm for Testing the Statistical Significance of Coherence Estimates}

Significance tests for the conventional coherence estimate [12] are well established [15], but they cannot be readily adapted for the case of missing samples, and the algorithm described above. Furthermore, in the current application, we do not expect the maximum coherence to necessarily occur at some previously precisely known frequency, but at any point within the frequency band corresponding to TA EEG activity. We, therefore, wish to test the significance of the maximum value of coherence in a given frequency band, and not just the coherence at any given frequency (or a set of frequencies). The use of repeated significance tests (one for each frequency component) is not recommended, as the probability of achieving a positive result increases, if the frequency range under investigation is expanded. This problem is common when applying multiple statistical tests, and the Bonferroni correction is one solution that is frequently applied. However, this solution cannot be employed in the current problem, because the coherence estimates are not independent. Following our previous approach [8], we therefore developed a solution using the Monte Carlo method, which can deal with the twin problem of 1) testing the significance of our modified coherence estimator (i.e., with missing samples), and 2) assessing the significance of the maximum coherence within a given frequency range.

In the Monte Carlo method [16], [17], random signals are generated, whose statistical characteristics reflect those of the data recorded from the subjects, and are known to obey the null-hypothesis, which in our case is $\mathrm{H} 0: C(f)=0$. We, thus, generate a set of $K$ pairs of uncorrelated random signals $\left(x_{k}^{*}[i]\right.$ and $y_{k}^{*}[i], k=1, \ldots, K,{ }^{*}$ indicating simulated signals), with the same pattern of missing samples, and the same respective power spectra as the recorded data. In the current work, we chose $K=499$ [16] simulated signals. For each of these signalpairs, the coherence is then estimated $\left(\left|\hat{C}_{k}^{*}(f)\right|\right)$, using the algorithm described above. This process provides a distribution of coherence estimates for uncorrelated signals, which reflects the sampling distribution of the estimator under the null-hypothesis of $C(f)=0$, and is illustrated in Fig. 2. In accordance with the percentile method [16], the percentage of coherence estimates from the Monte Carlo simulations that lie above the coherence obtained from the recorded data (marked at o and $*$ for two different frequencies in the figures) gives the significance (p-value) of the coherence estimate. If this percentage lies below the chosen significance level $(\alpha)$, the coherence estimate is considered statistically significant. The same approach is also applied in testing the significance of the maximum coherence value in a selected frequency band $\left(\max \{|\hat{C}(f)|\}, f_{1} \leq f \leq\right.$ $f_{2}$ ). For this reason, the cumulative density function of the maximum of $\left|\hat{C}_{k}^{*}(f)\right|$ in the selected frequency range is employed, rather than the coherence estimates at an individual frequency component.

There are different options in generating the random signals (also known as surrogate data) in this Monte Carlo method. If it may be assumed that the signals are zero-mean and Gaussian, their joint probability-density-functions are fully defined by their second-order statistics [13]. Thus, the surrogate signals should have the same power-spectrum as the recorded signals, and according to the null-hypothesis, they must of course also be uncorrelated. In previous work [8], we obtained these signals by using the amplitude spectra of the recorded data and by generating random phase spectra. In the present application, 

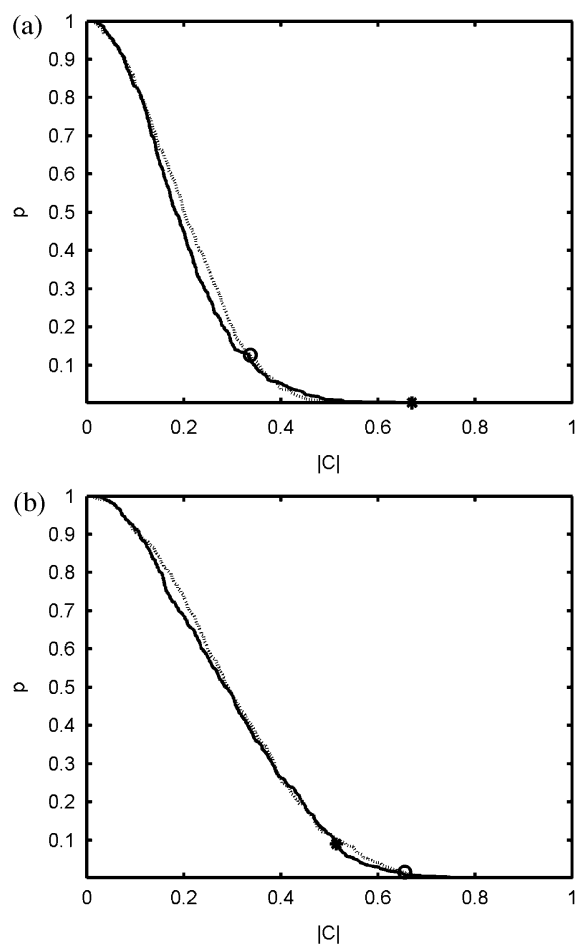

Fig. 2. Results from Monte Carlo simulations: The probability of obtaining coherence estimates greater than or equal to $|C|$ is shown for two selected frequencies $(0.094 \mathrm{~Hz}$ - solid line; $0.156 \mathrm{~Hz}$-dashed line) and two different recordings: (a) recording 4(2) and (b) recording 9 (see Table I). The coherence estimates from the signals recorded from the subjects' original signal are shown as $*$ for $0.094 \mathrm{~Hz}$, and o for $0.156 \mathrm{~Hz}$. In (a), this was larger than any estimates from the simulated data at $0.094 \mathrm{~Hz}(*)$ and, thus, it is considered highly significant ( $\mathrm{p}<0.002$, for 499 simulated signals $)$, whereas at $0.156 \mathrm{~Hz}$, some $12 \%$ of simulation results exceed the value ( $\mathrm{p}=0.12$-not significant). In (b), $\mathrm{p}=0.02$ and $\mathrm{p}=0.07$, respectively. The signal in (b) was shorter than in (a), which leads to the wider scatter in coherence estimates in the Monte Carlo simulation. Thus, for example, a coherence estimate of 0.5 would be significant $(\mathrm{p}<0.05)$ in (a) but not in (b).

we used an autoregressive (AR) model (order $p_{\mathrm{AR}}$ ). The autoregressive approach was also recommended in recent work [17] on coherence estimates. We estimated the coefficients of the model by applying the Yule-Walker method to the recorded data, using the autocorrelation functions $\left(\hat{R}_{x x}[m]\right.$ and $\hat{R}_{y y}[m]$ ) obtained according to (3). A triangular lag-window (as is commonly used in AR spectral estimates) was applied here (equivalent to the biased autocorrelation estimate, as recommended in [18]). The surrogate data were then obtained by filtering white Gaussian noise with this AR filter. Care was taken to avoid transients at the beginning of the signals $x_{k}^{*}[i]$ and $y_{k}^{*}[i]$, by progressively increasing the model order over the first $p_{\mathrm{AR}}$ samples (following the approach in [18]).

\section{RESULTS}

The coherence estimator and the Monte Carlo statistical tests were first assessed using simulated signals. The example used also serves to illustrate the procedures. Five hundred pairs of signals with known coherence between them were generated, from filtered white noise (filter coefficients $-0.283,-0.114,0.533,0.533,-0.114,-0.283$, and $1,-1.061,0.563$, for the numerator and denominator polynomials of the transfer function, respectively) and additive

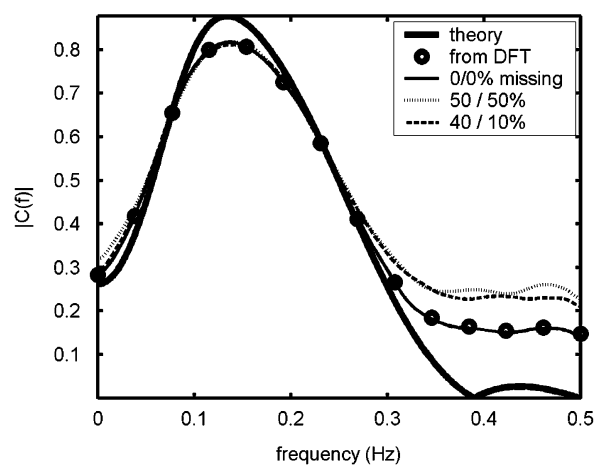

Fig. 3. Mean of coherence estimates from 500 pairs of simulated signals. The correct coherence for this example (as obtained from theory) is shown in bold. With no missing data in either $x[i]$ or $y[i]$, the estimates from overlapped FFTs (o) and the proposed algorithm (solid line) are almost identical. When samples are missing (50\% in $x[i]$ and $y[i]$, and $40 \%$ in $x[i]$ and $10 \%$ in $y[i]$, respectively), the bias error becomes slightly worse, but broadly correct estimates are still obtained.

low-pass-filtered noise. The example was chosen to give a wide range of coherence values and show overall characteristics that broadly approximate those from the neonatal data. A range of frequencies where coherence is very low was also included in the example. The simulated signals corresponded to a sampling rate of $f_{s}=1 \mathrm{~Hz}$. The results (Fig. 3) obtained with the new algorithm (mean of 500 simulations) are almost identical to those from the conventional coherence estimator (using overlapped FFTs; marked as o in Fig. 3). The latter could of course only be applied in the case of no missing samples, and they were obtained with a window-length of 26 samples (equivalent to the maximum lag of \pm 25 samples in the alternative estimator), an overlap of 25 samples, and Hanning windows. The mean of the coherence estimates (Fig. 3) approximates the true values (obtained from theory, using the filter coefficients and the noise levels selected for this simulation), although there is considerable bias, especially at the peak and where coherence is zero. Conventional coherence estimates are known to be biased [15], [19], and the proposed new algorithm does not overcome this limitation (nor was it expected to). When 50\% of samples of $x[i]$ and $y[i]$ were set as missing (dotted line) and then $40 \%$ of $x[i]$ and $10 \%$ of $y[i]$ (dashed line), the mean coherence estimates show a slight increase in bias error. As now less data are available for processing, this is not surprising. The signals were all 400 samples in length. For the case of $50 \%$ missing samples, these were disposed in two blocks of 121 and 81 samples, respectively, placed in identical locations in both signals. The $40 \%$ missing samples correspond to two blocks of 61 and 101 samples, respectively. The $10 \%$ of missing samples are contained in a single block, which partly overlaps the missing samples in the first signal.

The Monte Carlo test for the significance of the coherence estimate was then applied $(K=499)$ in each of the 500 simulated signal pairs. Thus, for each signal pair, the statistical significance (p-value) at each frequency component was obtained; when $p<\alpha=5 \%$, the coherence at that frequency was considered to be statistically significant. The results (Fig. 4) show that, as expected, statistically significant coherence is detected in most signal-pairs at frequencies where coherence is high (below frequencies of $0.35 \mathrm{~Hz}$ ). Where $|C(f)| \approx 0$, approximately $5 \%$ of cases are found to be significant (thin hor- 




Fig. 4. The fraction of cases (in 500 simulations; see Fig. 3) in which the Monte Carlo test showed statistically significant coherence (at a significance level $\alpha=5 \%$ ) between pairs of signals. Where coherence is high, all simulated signals gave significant results. Where $|C(f)|=0$, the expected false-positive rate of 5\% (shown as a thin horizontal line) was obtained. As the number of missing samples increases (dotted and dashed lines), statistical significance is obtained in fewer cases, but the false-positive rate is maintained at approximately the expected level.

izontal line), which is in accordance with the selected significance level of $\alpha=5 \%$. The Monte Carlo significance tests of coherence gave similar results to those obtained by the conventional method based on the normalizing transform [19], in the case where the latter can be applied, i.e., when no data were missing (solid line). In the signals with missing samples (dotted and dashed lines), fewer cases are statistically significant where coherence is high, but the false-positive rate (above about 0.35 $\mathrm{Hz})$ remains approximately correct $(\hat{\alpha} \approx 5 \%)$.

To check the false-positive rate further, we applied the technique to pairs of independent signals. To this end, we generated 500 pairs of independent white noise signals, and then applied the same filters used in the previous example. Thus, the signals $x[i]$ and $y[i]$ had the same power spectra as before, but a theoretical coherence of zero. We then estimated the coherence between these signals as before, and we tested the statistical significance of the estimates. The results showed the false-positive rate to be close to the expected value of $\alpha=5 \%$ in signals with and without missing samples, for all frequencies. When we considered the maximum coherence in a band of frequencies from 0.08 to $0.13 \mathrm{~Hz}$ (the same range of interest as used in the data recorded from our subjects), the Monte Carlo test described above gave close to the expected $\alpha=5 \%$ false-positive rate, both with and without missing samples. The need for a specific test for maximum coherence is evident from the observation that $12 \%$ of the 500 signal pairs showed at least one statistically significant coherence estimate (at $\alpha=5 \%$ ) in the same frequency range.

The coherence estimator was then applied to mCBFV and rmsEEG signals recorded from the neonates. The median and quartiles of the coherence estimates [Fig. 5(a)] from the 12 recordings show that there is a clear peak in coherence at around $0.1 \mathrm{~Hz}$, with the median value reaching 0.59 . The p-values [Fig. 5(b)] show a clear minimum in the same frequency band, where the median value drops below $\mathrm{p}=5 \%$.

The results presented were obtained with autoregressive model orders of 10. We also applied the Akaike Information Criterion (AIC) on each signal to estimate optimal orders. The minimum of the AIC indicated orders between 1 and 14
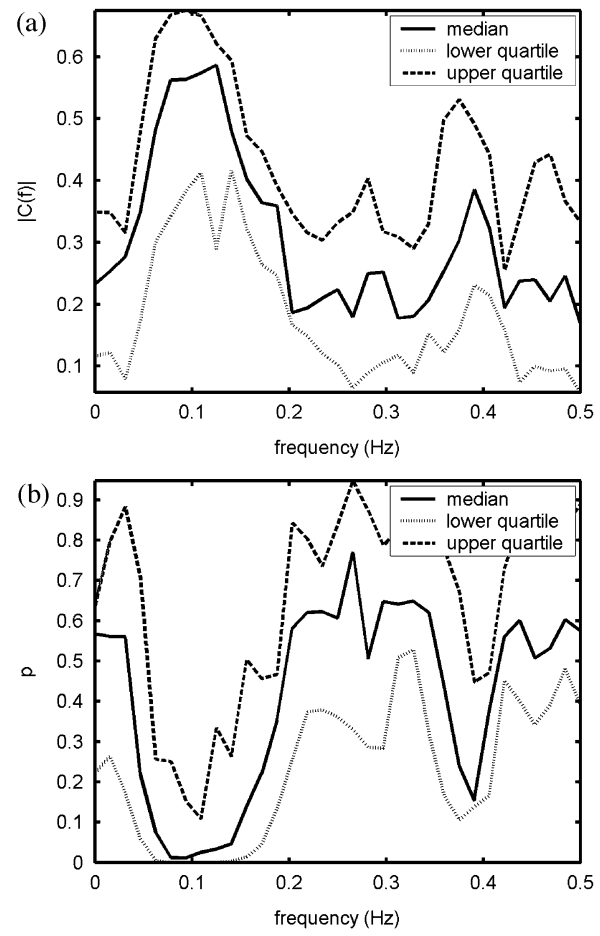

Fig. 5. The $|C(f)|$ (a) for the set of 12 signals studied, together with the corresponding p-values (b).

for the signals (mostly below 10). However, AIC and similar criteria are known often to underestimate the order of "real" (as opposed to simulated) data [13], and the presence of multiple (and shallow) minima in the AIC function suggested that a considerable range of model orders are almost equally as good. We, thus, repeated the Monte Carlo simulation with constant orders of 5,10, and 14, as well as the "optimal" order for all signals, and results were all similar. Results presented in this paper are those for model order 10.

Considering the broad peak around $0.1 \mathrm{~Hz}$, which varies between individuals, we tested the maximum coherence in the range from 0.08 to $0.13 \mathrm{~Hz}$. It was found that in 10 of the 12 recordings (from eight of the nine subjects), at least one of the frequencies in this range gave statistically significant coherence. When applying the Monte Carlo test for the maximum coherence in this frequency range, 8 of the 12 recordings (from six of the nine subjects) were found to have a statistically significant peak in coherence (Table I). In two more subjects, significance was almost attained $(\mathrm{p}<7 \%)$.

To consider the coherence in the context of the power spectra of the signals, the median and quartiles of the power spectra for mCBFV and rmsEEG are shown in Fig. 6(a) and (b), respectively. The latter have a spectral peak at around $0.1 \mathrm{~Hz}$ (as was expected from the usual periodicity of TA EEG), whereas the former contain predominantly lower frequencies.

\section{DISCUSSION}

In previous work [8], we had found that there is low, but statistically significant cross-correlation between mean CBFV and the power of the EEG signal during TA activity in the newborn. There are sound physiological reasons why such correlation might be expected, given our understanding of cerebral 



Fig. 6. The power-spectral density (obtained from autoregressive modeling and order 10) of the mCBFV (a) and rmsEEG (b). The median (solid line) and quartiles (dotted lines) for the 12 recordings are shown. The power-spectral densities are given in units of $\frac{(\mathrm{cm} / \mathrm{s})^{2}}{\mathrm{~Hz}}$ and $\frac{\mu \mathrm{V}^{2}}{\mathrm{~Hz}}$, respectively.

blood flow control [1]. Even in premature babies, evidence of active blood flow control has been found, and this progressively strengthens with gestational age [20]. However, we had been unsure if a relationship between EEG and CBFV would be evident in spontaneous EEG activity of neonates. With the current work, we aimed to refine our previous results, by ascertaining in which frequency band the dependence between the signals is strongest. The coherence function is the obvious tool for this purpose, and it provided a clear answer to the question: Coherence is strongest in a frequency band around $0.1 \mathrm{~Hz}$, during TA EEG activity. Only one of the nine subjects (both recordings of subject 1 , Table I) gave clearly nonsignificant results. Two further subjects were just above the $\alpha=5 \%$ threshold; it may be noted that both were relatively short, and statistically significant results were, therefore, more difficult to achieve. The frequency of the coherence peak corresponds roughly to the periodicity of the TA pattern, where high- and low-voltage activity alternates in approximately 10 -s cycles. The power spectra of rmsEEG showed a pronounced peak in the vicinity of $0.1 \mathrm{~Hz}$ in all cases; such peaks were less evident (although often present) in mCBFV.

The ability of the cerebral vasculature to respond to variations in the demand for blood supply is of great clinical relevance, as several serious neonatal pathologies have been linked to failings of the blood flow control system [21]-[23]. A wide range of methods have been developed to assess cerebral blood flow control, based on transcranial Doppler measurements of blood flow and recordings of blood pressure [24], but none has proven entirely satisfactory for routine clinical application in neonates. One reason for this difficulty is that cerebral blood flow control involves complex mechanisms, which are affected by many fac- tors [4], [25]-[27]. The current work further demonstrates that spontaneous EEG activity is significantly correlated with CBFV, and this may need to be considered in mathematical models of blood flow control. It is interesting to note that the frequency range around $0.1 \mathrm{~Hz}$, where highest correlation was found in the current work, was also found to be particularly relevant in other studies of cerebral blood flow control, which did not involve the EEG signal [24], [28].

The main benefit of the novel algorithm proposed for estimating coherence is that it can be applied in signals where some samples are missing, and it provides a simple means of testing the statistical significance of the results. The intermittent loss of data is a common problem in signals acquired from human subjects, where noise, artifact, and variations in physiological state often lead to interrupted recordings.

The results from simulation studies provide validation of the method presented. Coherence estimates are close to the expected values and, without missing samples, give results similar to those obtained with the conventional technique [12]. Recent work [17] also advocated the use of surrogate data to study coherence estimates. In that work, the failure of conventional statistical techniques in the presence of sharp spectral peaks was considered, and neither missing data, nor maximal coherence over a band of frequencies, were investigated. In some of our earlier simulation studies, problems were encountered with coherence estimates that occasionally exceeded unity: This occurred where true coherence values were close to one in the immediate vicinity (in a range corresponding roughly to the bandwidth of the lag-window) of rapid variations in signal spectra. This problem may be explained considering spectral leakage, which can greatly distort spectral estimates, and even lead to negative values in the power-spectral-density estimates from the correlogram method [13]. The use of the Hanning window (convolved with itself for the lag-window) can resolve this problem for spectral estimates [13], and in the current work on signals with missing samples, it was also found to greatly reduce the incidence of excessive coherence values. Even so, near sharp transients in the power spectra, coherence estimates may occasionally exceed unity, and they are, thus, clearly invalid. With the human data investigated here, this problem did not arise, and in no cases were excessive coherence values obtained.

Care has to be taken in applying the proposed method, in regard to the length of data available. The maximum lag is inversely proportional to the frequency resolution of coherence estimates, which makes longer lags desirable. For signals without missing samples, it has been noted that the maximum lag in estimates of autocorrelation (and cross-correlation) should be much smaller than the length of the signals available [13], to avoid excessive estimation errors. These considerations should also be applied in signals with some missing samples, where the number of samples that can be used at each lag $[L$ in (3)] depends not only on the lag, but also on the distribution of missing samples. If at any lag $L$ falls to zero, the estimator fails.

In common with other algorithms for estimating coherence, stationarity and ergodicity of the signals is assumed. As in most biomedical applications, this assumption is probably not strictly valid. High-pass filtering was used to greatly attenuate trends and low-frequency variations that are probably the most 
common causes of nonstationarity in bioelectric signals. In addition, by removing signal segments containing evident artifact, and selecting only those segments containing the TA EEG pattern, stationarity is probably better approximated than in some other applications. Detailed analysis of the effect any nonstationarity may have on the coherence analysis is beyond the scope of this paper.

The selection of the autoregressive model order is potentially a problem in this method, as the usual techniques are generally known to be unreliable [13]. However, the results we obtained suggested that the estimated p-values are not sensitive to the signals accurately simulating the spectrum of the original data. This is in agreement with the conventional techniques for the statistical analysis of coherence estimates [15], in which the number of data-windows and the true coherence determines the random error in the estimates, but the signals' spectra are not determining factors. More accurate modeling of the signals does, however, seem to become important when the spectra contain sharp peaks [17], which is not the case in our data.

Computational load for the Monte Carlo simulations was not excessive: Each signal pair required some $5 \mathrm{~s}$ to perform the Monte Carlo significance tests, using Matlab ${ }^{\circledR}$ on a Pentium 4 processor. Considerable improvements in speed could probably be achieved by more efficient implementations of the proposed algorithm.

Although the technique was effective in solving the original problem of estimating the coherence between CBFV and EEG activity, we hope the algorithm will also provide a powerful tool for a much wider range of applications in biomedical signal processing. The results obtained provide the basis for our continuing investigation of cerebral blood flow control. One main question to be addressed now is whether the observed variations in $\mathrm{CFV}$ are mediated through concomitant changes in blood pressure (or other physiological variables), or whether they reflect active cerebrovascular blood flow control.

\section{ACKNOWLEDGMENT}

This work was carried out within the ongoing collaborative project Neonatal cerebral blood flow: Physiological control mechanisms and patient management (Wellcome Trust, U.K.). The authors thank Prof. D. Evans and Dr. L. Fan, Department of Medical Physics, Leicester Royal Infirmary, Leicester, U.K., for the development and loan of the signal acquisition system used.

\section{REFERENCES}

[1] H. C. Lou, L. Edvinsson, and E. T. MacKenzie, "The concept of coupling blood flow to brain function: Revision required?," Ann. Neurol., vol. 22, no. 3, pp. 289-297, 1987.

[2] P. Sandor, "Nervous control of the cerebrovascular system: Doubts and facts," Neurochem. Int., vol. 35, no. 3, pp. 237-259, 1999.

[3] G. Hajak, J. Klingelhöfer, M. Schulz-Varszegi, G. Matzander, D. Sander, B. Conrad, and E. Rüther, "Relationship between cerebral blood flow velocities and cerebral electrical activity in sleep," Sleep, vol. 17, no. 1, pp. 11-19, 1994

[4] F. Ferrari, A. W. R. Kelsall, J. M. Rennie, and D. H. Evans, "The relationship between cerebral blood flow velocity fluctuations and sleep state in normal newborns," Pediatr. Res., vol. 33, no. 1, pp. 50-54, 1994.

[5] J. M. Perlman and J. J. Volpe, "Seizures in the preterm infant: Effects on cerebral blood flow velocity intracranial pressure, and arterial blood flow," J. Pediatr., vol. 102, no. 2, pp. 288-293, 1983.
[6] G. B. Boylan, R. B. Panerai, J. M. Rennie, D. H. Evans, S. Rabe-Hesketh, and C. D. Binnie, "Cerebral blood flow velocity during neonatal seizures," Arch. Dis. Child., vol. 80, pp. F105-F110, 1999.

[7] R. Aaslid, "Visually evoked dynamic blood flow response of the human cerebral circulation," Stroke, vol. 18, pp. 771-775, 1987.

[8] D. M. Simpson, A. F. C. Infantosi, and D. A. B. Rosas, "Estimation and significance testing of cross-correlation between cerebral blood flow velocity and background electro-encephalograph activity in signals with missing samples," Med. Biol. Eng. Comput., vol. 39, pp. 428-433, 2001.

[9] A. J. Isaksson, "Identification of ARX-models subject to missing data," IEEE Trans. Automat. Contr., vol. 38, no. 5, pp. 813-819, May 1993.

[10] R. Pintelon and J. Schoukens, "Frequency domain system identification with missing data," IEEE Trans. Automat. Contr., vol. 45, no. 2, pp. 364-369, Feb. 2000.

[11] R. Ruf, "The lomb-scargle periodogram in biological rhythm research: Analysis of incomplete and unequally spaced time-series," Biol. Rhythm Res., vol. 30, no. 2, pp. 178-201, 1999.

[12] G. C. Carter, C. H. Knapp, and A. H. Nuttall, "Estimation of the magnitude-squared coherence function via overlapped fast Fourier transform processing," IEEE Trans. Audio Electroacoust., vol. AU-21, pp. 337-344, Apr. 1973.

[13] S. L. Marple, Digital Spectral Analysis with Applications. . Englewood Cliffs, NJ: Prentice Hall, 1987.

[14] P. D. Welch, "The use of fast Fourier transform for the estimation of power spectra: A method based on time averaging over short modified periodograms," IEEE Trans. Audio Electroacoust., vol. AU-15, pp. 70-73, Feb. 1967.

[15] V. A. Benignus, "Estimation of the coherence spectrum and its confidence interval using the fast Fourier transform," IEEE Trans. Audio Electroacoust., vol. AU-17, pp. 145-150, Feb. 1969.

[16] B. F. J. Manly, Randomization, Bootstrap and Monte Carlo Methods in Biology. London, U.K.: Chapman \& Hall, 1997.

[17] L. Faes, G. D. Pinna, A. Porta, R. Maestri, and G. Nollo, "Surrogate data analysis for assessing the significance of the coherence function," IEEE Trans. Biomed. Eng., vol. 51, no. 7, pp. 1156-1166, Jul. 2004.

[18] S. M. Kay, Modern Spectral Estimation: Theory and Application. Englewood Cliffs, NJ: Prentice-Hall, 1988, pp. 1-543.

[19] R. K. Otnes and L. Enochson, Applied Time Series Analysis. New York: Wiley, 1978. Basic Techniques.

[20] P. K. Verma, R. B. Panerai, J. M. Rennie, and D. H. Evans, "Grading of cerebral autoregulation in preterm and term neonates," Pediatr. Neurol., vol. 23 , pp. $236-242,2000$

[21] G. Jorch and N. Jorch, "Failure of autoregulation of cerebral blood flow in neonates studied by pulsed Doppler ultrasound of the internal carotid artery," Eur. J. Pediatr., vol. 146, pp. 468-472, 1987.

[22] H. C. Lou, N. A. Lassen, and B. Friis-Hansen, "Impaired autoregulation of cerebral blood flow in the distressed newborn infant," J. Pediatr., vol. 94, no. 1, pp. 118-121, 1979.

[23] D. W. A. Milligan, "Failure of autoregulation and intraventricular haemorrhage in preterm infants," Lancet, vol. i, pp. 896-898, 1980.

[24] R. B. Panerai, "Assessment of cerebral pressure autoregulation in humans-A review of measurement methods," Phys. Meas., vol. 19, pp. 305-338, 1998.

[25] M. Ursino, C. A. Lodi, and G. Russo, "Cerebral hemodynamic response to $\mathrm{CO} 2$ tests in patients with internal carotid artery occlusion: Modeling study and in vivo validation," J. Vasc. Res., vol. 37, pp. 123-133, 2000.

[26] G. Jorch, T. Huster, and H. Rabe, "Dependency of Doppler parameters in the anterior cerebral artery on the behavioral states in term and preterm neonates," Biol. Neonate, vol. 58, pp. 79-86, 1990.

[27] D. M. Simpson, R. B. Panerai, D. H. Evans, J. Garnham, A. R. Naylor, and P. R. F. Bell, "Estimating normal and pathological dynamic responses in cerebral blood flow velocity to step changes in end-tidal pCO2," Med. Biol. Eng. Comput., vol. 38, pp. 535-539, 2000.

[28] D. M. Simpson, R. B. Panerai, D. H. Evans, and A. R. Naylor, "A parametric approach to measuring cerebral blood flow autoregulation from spontaneous variations in blood pressure," Ann. Biomed. Eng., vol. 29, pp. 18-25, 2001.

David M. Simpson (M'95) photograph and biography not available at the time of publication.

Daniel A. Botero Rosas, photograph and biography not available at the time of publication.

Antonio Fernando C. Infantosi, photograph and biography not available at the time of publication. 